\title{
Derivation of Shrinking Core Model for Finite Cylinder
}

\author{
Jeremy MOON and Veena SAHAJWALLA \\ School of Materials Science and Engineering, University of New South Wales, Sydney 2052 NSW Australia.
}

(Received on May 18, 2000; accepted in final form on October 13, 2000)

\begin{abstract}
A model has been developed to describe the shrinking core style conversion of a solid finite cylinder by gas. The model describes the three limiting cases of gas film diffusion, product layer diffusion and chemical reaction controlled kinetics. The model is validated with experimental data obtained from literature. Rate constants obtained are similar to those from literature.
\end{abstract}

KEY WORDS: mathematical modelling; kinetics; shrinking core model; finite cylinder; reduction.

\section{Introduction}

The effect of geometry on shrinking core solid-fluid kinetics has been studied for some time, with the development of mathematical models for both the radial (sphere and infinite cylinder) and Cartesian (flat plate) co-ordinate systems. These models are summarised by Levenspiel. ${ }^{1)}$ The solutions to these geometries are elegant and easily usable and are based on the minimum of assumptions. However, when using geometries other than these three, complications arise on being able to describe the movement of the shrinking boundary in terms of one of the co-ordinate systems (or being able to describe them at all) while still keeping to a minimum of assumptions leading to near impossible situations. Accordingly, attempts to obtain workable models for systems other than the above three have meant the adoption of assumptions. Two attempts at describing a finite cylinder provide examples of this point.

Firstly Gorkan and Doraiswamy ${ }^{2)}$ attempted to provide a solution for the most difficult of scenarios with the finite cylinder, that of product layer diffusion controlled. For the product layer diffusion description, two integration steps are required. The first integration step describes the rate of diffusion of the reacting species through the product layer to the reacting interface. This is achieved by describing the instantaneous concentration profile (with respect to the cross sectional area) of diffusing species within the sample at any given time $(t)$. The second integration step equates the rate of diffusion into the sample with the rate of reaction of the reacting solid. As this is a shrinking core model, this step is described in geometric terms. That is, the description of the reaction interface movement with time in terms of the chosen co-ordinate system.

Gorkan and Doraiswamy ${ }^{2)}$ simplified their situation by choosing to circumvent the first integration step, instead using an averaged cross sectional flux area to determine the instantaneous diffusion rate. In order to achieve this, they had to approximate the movement of the un-reacted core height with degree of reaction. Their solution was to assume that the height change was proportional to the radius change. That is, the produced product layer was of uniform thickness over the cylinder.

The flaw in this assumption is illustrated by breaking up a finite cylinder into components whose descriptions have been well established. The flat plate (describing the ends of the cylinder) and the infinite cylinder (describing the radial portion of the cylinder). For the flat plate, the cross sectional flux area remains constant, and therefore the diffusion rate in a flat plate is constant. For an infinite cylinder, as the cross sectional flux area decreases with radius, and therefore the diffusion rate through the outer surface of the radial surface also decreases. Accordingly, it can be seen that the product layer on the ends of a finite cylinder will develop at a different rate to the radial portion.

Kang et al. ${ }^{3)}$ have worked on the extremely difficult task of providing a workable mathematical model of generic non-spherical systems. In doing so, they developed two models. The first uses an approximation similar to that of Gorkan and Doraiswamy, ${ }^{2)}$ in that the reaction interface proceeds at the same rate. In other words the product layer is uniform over the entire sample. As explained above, it is seen that this is flawed when applied to the geometry of a finite cylinder.

The second model uses an assumption that the relative product layer thickness for any direction is the same. That is, the product layer would reach the geometric centre of a sample from all directions at the same time. This assumption also has difficulties when applied to a finite cylinder. To illustrate the flaw, two hypothetical finite cylinders of different heights are used. In order for the product layer to reach the geometric centre of each cylinder at the same time, either the diffusivity through the ends of the taller finite cylinder would have to be higher than the diffusivity of the shorter finite cylinder or the diffusivity through the radial portion of the taller finite cylinder would have to be lower than the shorter finite cylinder. Since the diffusivity is a material constant and not a geometric constant, it can be seen that the assumption is not valid.

This paper aims to provide methods to solve the shrinking core kinetic models (namely gas film diffusion, chemi- 
Table 1. Kang et al. sample description. ${ }^{3)}$

\begin{tabular}{|c|c|c|c|c|}
\hline Temperature & Sample ID & Diameter $(\mathrm{cm})$ & Height $(\mathrm{cm})$ & Mass $(\mathrm{g})$ \\
\hline $800^{\circ} \mathrm{C}$ & P2 & 1.19 & 0.9 & 4 \\
\hline $800^{\circ} \mathrm{C}$ & P5 & 1.66 & 0.92 & 8 \\
\hline $900^{\circ} \mathrm{C}$ & P5 & 1.66 & 0.92 & 8 \\
\hline
\end{tabular}

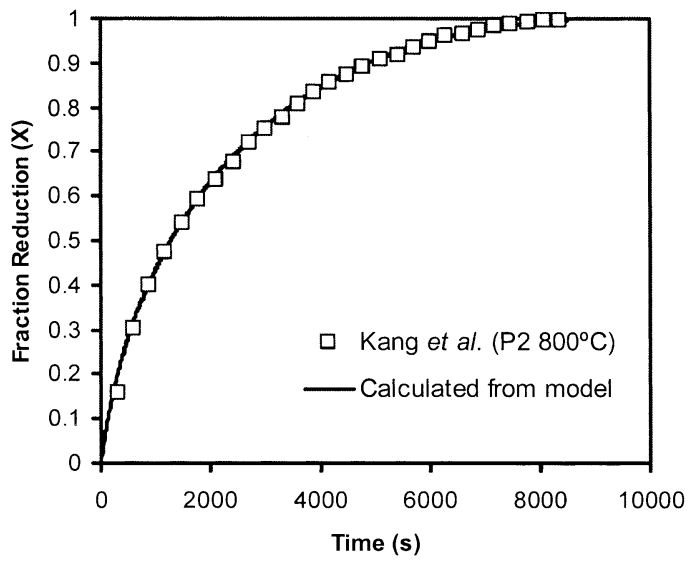

Fig. 1. Reduction data extracted from Kang. ${ }^{3)} \mathrm{P} 2-800^{\circ} \mathrm{C}$ (see Table 1) along with data calculated from the model. Note that for this experiment, $X=0$ when all the sample is $\mathrm{Fe}_{2} \mathrm{O}_{3}$ and $X=1$ when all the sample is $\mathrm{Fe}_{3} \mathrm{O}_{4}$.

cal reaction and product layer diffusion controlled) for the finite cylinder. The models will be checked against experimental data obtained by Kang et al. ${ }^{3)}$ and Nasr et al., ${ }^{4)}$ both of whom have conducted gas-solid reaction experiments on finite cylinders.

\section{Experimental}

Data from literature was extracted and used to examine the validity of the developed models. The data used is that of Kang et al. ${ }^{3)}$ and Nasr et al. ${ }^{4)}$

\subsection{Kang et al.'s ${ }^{3)}$ Experimental Method}

The experimental method was described in their earlier paper, ${ }^{5)}$ Amongst other shapes, reduction of haematite finite cylinders was conducted at 800 and $900^{\circ} \mathrm{C}$. The $99 \%$ pure $\mathrm{Fe}_{2} \mathrm{O}_{3}$ was reduced to $\mathrm{Fe}_{3} \mathrm{O}_{4}$ in a thermo-gravimetric apparatus using a $\mathrm{CO}: \mathrm{CO}_{2}$ mixture of $10: 90$. Of particular note, the experiments were designed to minimise the effect of gas film diffusion on the overall reduction process. Description of their samples is given in Table 1. ${ }^{3)}$ Data extracted from their paper ${ }^{3)}$ is shown in Fig. 1 to Fig. 3.

\subsection{Nasr et al.'s ${ }^{4)}$ Experimental Method}

The experimental method was described in their paper. ${ }^{4)}$ Reduction of haematite finite cylinders (compacts) was conducted from 700 to $1100^{\circ} \mathrm{C} .97 .82 \%$ pure $\mathrm{Fe}_{2} \mathrm{O}_{3}$ finite cylinders $(0.79 \mathrm{~cm}$ (diameter $) \times 0.835 \mathrm{~cm}$ (height) $)$ were reduced to Fe using CO. Data extracted from Nasr et al.'s paper $^{4)}$ is shown in Fig. 4.

\section{Theory}

The main difficulty in approaching the cylinder over the sphere is that where the sphere possesses a single (radial)

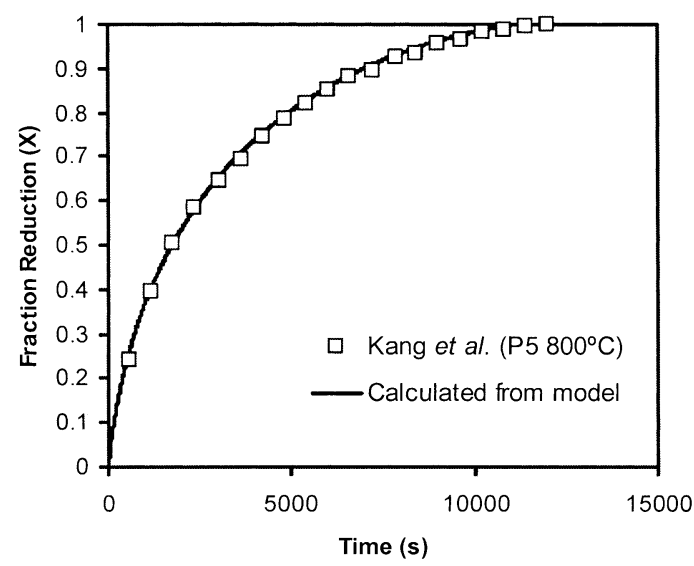

Fig. 2. Reduction data extracted from Kang. ${ }^{3)} \mathrm{P} 5-800^{\circ} \mathrm{C}$ (see Table 1) along with data calculated from the model. Note that for this experiment, $X=0$ when all the sample is $\mathrm{Fe}_{2} \mathrm{O}_{3}$ and $X=1$ when all the sample is $\mathrm{Fe}_{3} \mathrm{O}_{4}$.

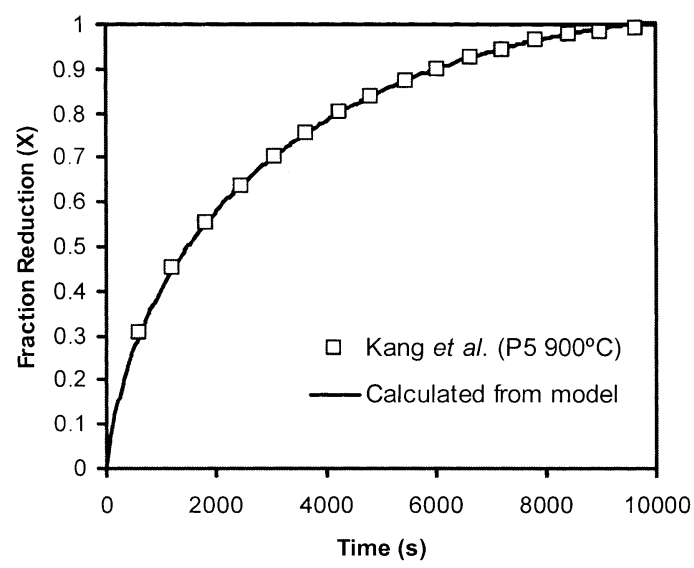

Fig. 3. Reduction data extracted from Kang. ${ }^{3)} \mathrm{P} 5-900^{\circ} \mathrm{C}$ (see Table 1) along with data calculated from the model. Note that for this experiment, $X=0$ when all the sample is $\mathrm{Fe}_{2} \mathrm{O}_{3}$ and $X=1$ when all the sample is $\mathrm{Fe}_{3} \mathrm{O}_{4}$.

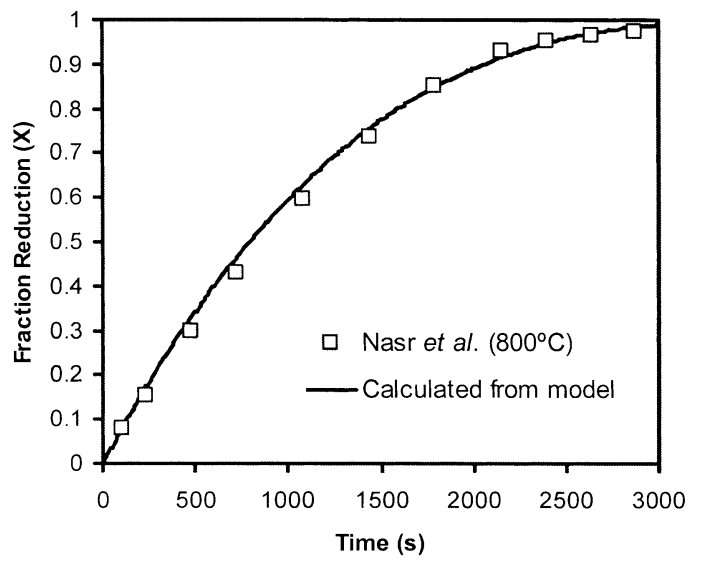

Fig. 4. Reduction data extracted from Nasr et al. ${ }^{4)} 800^{\circ} \mathrm{C}$ along with data calculated from the model. Note that for this experiment, $X=0$ when all the sample is $\mathrm{Fe}_{2} \mathrm{O}_{3}$ and $X=1$ when all the sample is Fe.

co-ordinate system, the cylinder comprises of both a radial and Cartesian co-ordinate system as shown in Fig. 5. This makes the integration of flux surface areas difficult, but this can be overcome by the description of the Cartesian co-ordinates in terms of the radial co-ordinate system. This re- 
Z

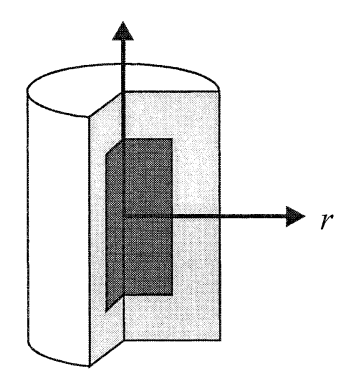

a) b)

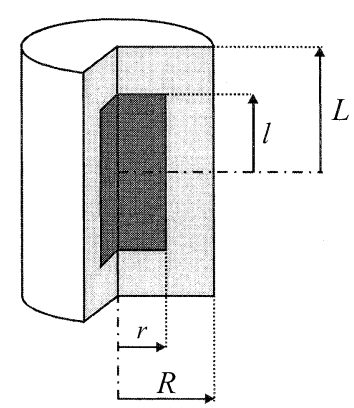

Fig. 5. a) Schematic representation of finite cylinder showing the combination of radial co-ordinates $(r)$ and Cartesian co-ordinates $(z)$. b) Schematic representation of finite cylinder with $R$ and $r$ representing the distance from the axis to the radial outer surface and un-reacted core radial surface respectively. The values $L$ and $l$ represent the distance from the cylinder mid-height to the cylinder end and un-reacted core end respectively.

placement depends on the specific system being studied.

In the following cases, a radial description of the Cartesian co-ordinates is obtained before applying it to the shrinking core models.

\subsection{Gas Film Diffusion}

3.1.1. Description of Cylinder Height in Terms of Radius

To describe the progression of the end of a cylinder with respect to the radius of the cylinder, we can employ the individual equations describing the conversion of a flat plate (end of cylinder), and that of the infinite cylinder (radial section of the cylinder). As there exists equations ${ }^{1)}$ which already describe the flat plate and the cylinder, we need a case which can link these two. This is achieved by coupling the total time required for conversion for each mode. To do this, we consider the reaction:

$$
\mathrm{A}_{(\mathrm{g})}+b \mathrm{~B}_{(\mathrm{s})} \rightarrow r \mathrm{R}_{(\mathrm{g})}+s \mathrm{~S}_{(\mathrm{s})}
$$

In the case of oxygen removal from iron, $B$ is the oxygen atom, and $b=1$. By using this equation, we can now determine which 'unique' finite cylinder height $\left(2 \cdot L_{\mathrm{u}}\right)$ and 'unique' cylinder radius $\left(2 \cdot R_{\mathrm{u}}\right)$ satisfy the case where the total time for conversion of an infinite cylinder equals the total time for conversion of a flat plate $\left(i . e .: \tau_{\text {i.c. }}=\tau_{\text {f.p. }}\right)$. The respective total times of conversion for each model is given as:

for an infinite cylinder (i.c.)

$$
\tau_{\text {i.c. }}=\frac{\rho_{\mathrm{B}} R_{\mathrm{u}}}{2 b k_{\mathrm{g}} C_{\mathrm{Ag}}}
$$

Where $b$ is the number of moles of $\mathrm{B}$ reacted with every mole of $\mathrm{A}, C_{\mathrm{Ag}}$ is the molar concentration of gas species $\mathrm{A}$ $\left(\mathrm{mol} / \mathrm{cm}^{3}\right), k_{\mathrm{g}}$ is the gas film diffusion constant and $R_{\mathrm{u}}$ is the 'unique' radius of finite cylinder (same as $R$ ).

and for a flat plate (f.p.)

$$
\tau_{\text {f.p. }}=\frac{\rho_{\mathrm{B}} L_{\mathrm{u}}}{b k_{\mathrm{g}} C_{\mathrm{Ag}}}
$$

Where $L_{\mathrm{u}}$ is the 'unique' half height of finite cylinder.

From this:

$$
L_{\mathrm{u}}=\frac{R_{\mathrm{u}}}{2}
$$

For this case, the fractional time of reduction $(t / \tau)$ is the same for each mode of conversion, and therefore a relationship for the change in cylinder height with radius can be determined. The fraction time of reduction for the flat plate and infinite cylinder are given by Levenspiel ${ }^{1)}$ as:

$$
\begin{gathered}
\left(\frac{t}{\tau_{\text {i.c. }}}\right)=1-\left(\frac{r}{R_{\mathrm{u}}}\right)^{2} \\
\left(\frac{t}{\tau_{\text {f.p. }}}\right)=1-\frac{l_{\mathrm{u}}}{L_{\mathrm{u}}} .
\end{gathered}
$$

Where $r$ is the radius of the finite cylinder core and $l_{\mathrm{u}}$ is the 'unique' half height of finite cylinder core.

Using Eqs. (4) and (5), it can be seen that at any time ( $t$ ):

$$
l_{\mathrm{u}}=L_{\mathrm{u}} \cdot\left(\frac{r}{R_{\mathrm{u}}}\right)^{2}
$$

For film layer diffusion controlled conversion, the in-flux of reactant gas through the outer surface area remains constant. However the change in reaction surface area for the radial section of the infinite cylinder decreases with degree of conversion, whereas the flat plate reaction area remains constant. Therefore we see that the geometric description given above satisfies this situation.

As the cylinder end acts independently to the radius, describing cases where $L \neq R / 2$, Eq. (6) can be transposed as follows.

$$
l=l_{\mathrm{u}}+L-L_{\mathrm{u}}=L_{\mathrm{u}}\left(\left(\frac{r}{R}\right)^{2}-1\right)+L
$$

On examining Eq. (7) we see that:

for $L>R / 2$

$$
\begin{aligned}
& \tau_{\text {f.p }}>\tau_{\text {i.c. }} \\
& \tau_{\text {cyl }}=\tau_{\text {i.c. }}
\end{aligned}
$$


and that for $L<R / 2$

$$
\begin{gathered}
\tau_{\text {f.p }}>\tau_{\text {i.c. }} \\
\tau_{\text {cyl }}=\tau_{\text {f.p. }}
\end{gathered}
$$

\subsubsection{Formulation Method of Model}

For gas film diffusion controlled mechanisms, the rate of conversion is proportional to the flux at the surface of the cylinder. It is assumed that the gas film is sufficiently thin that there is no change in radius for the concentration gradient across it. It is also assumed that the concentration of reactants at the surface of the cylinder is zero (as per Levenspiel $^{1)}$ ). Considering the reaction described in Eq. (1) we get:

$$
-\frac{d N_{\mathrm{B}}}{d t}=-b \frac{d N_{\mathrm{A}}}{d t}=b k_{\mathrm{g}} A_{\text {surface }} C_{\mathrm{Ag}}=2 \pi R(R+2 L) \cdot b k_{\mathrm{g}} C_{\mathrm{Ag}}
$$

Where $A_{\text {surface }}$ is the surface area of finite cylinder, $k_{\mathrm{g}}$ is the gas film diffusion constant and $N_{x}$ is the number of moles of species ' $x$ '.

It is possible to rewrite $N_{\mathrm{B}}$ in terms of the shrinking core.

$$
\begin{gathered}
-2 \pi r\left(r+2 L_{\mathrm{u}}\left(\left(\frac{r}{R}\right)^{2}-1\right)+2 L\right) \cdot \rho_{\mathrm{B}} \frac{d r}{d t} \\
=2 \pi R(R+2 L) \cdot b k_{\mathrm{g}} C_{\mathrm{Ag}} \ldots \ldots \ldots \ldots . . . \\
-\int_{R}^{r} r\left(r+2 L\left(\left(\frac{r}{R}\right)^{2}-1\right)+2 L\right) d r \\
=\frac{R(R+2 L) \cdot b k_{\mathrm{g}} C_{\mathrm{Ag}}}{\rho_{\mathrm{B}}} \int_{0}^{t} d t \ldots \ldots \ldots \ldots . . . \\
t \cdot \frac{b k_{\mathrm{g}} C_{\mathrm{Ag}}}{\rho_{\mathrm{B}}}\left(\frac{-r^{2}\left(3 L_{\mathrm{u}} r^{2}-6 L_{\mathrm{u}} R^{2}+6 L R^{2}+2 R^{2} r\right)}{6 R^{2}}\right. \\
=\frac{1}{R(R+2 L)}\left(\frac{L_{\mathrm{u}} R^{2}}{2}+L R^{2}+\frac{R^{2}}{3}\right) \ldots \ldots \ldots \ldots . . .
\end{gathered}
$$

Note: Firstly the radius can be determined explicitly from the degree of conversion $\left(X_{\mathrm{B}}\right)$ by the following correlation:

$$
\begin{aligned}
& X_{\mathrm{B}}=\frac{V_{\text {total }}-V_{\text {core }}}{V_{\text {total }}}=1-\frac{4 \pi \cdot r^{2}\left(L_{\mathrm{u}}\left(\left(\frac{r}{R}\right)^{2}-1\right)+L\right)}{4 \pi \cdot R^{2} L} \\
& =1-\frac{r^{2}\left(L_{\mathrm{u}}\left(\left(\frac{r}{R}\right)^{2}-1\right)+L\right)}{R^{2} L}
\end{aligned}
$$

The most geometrically appropriate root gives the radius of:

$$
\begin{aligned}
r= & \frac{1}{2} \cdot\left\{-\left(-2 R^{2} L_{\mathrm{u}}+2 R^{2} L\right.\right. \\
& \left.\left.-2 \cdot \sqrt{R^{4} L_{\mathrm{u}}^{2}+2 R^{4} L \cdot L_{\mathrm{u}}+R^{4} L^{2}-4 L_{\mathrm{u}} R^{4} L X_{\mathrm{B}}}\right) / L_{\mathrm{u}}\right\}^{1 / 2}
\end{aligned}
$$

and secondly, that for $L<R / 2$

$$
r \neq 0 \text { when } t=\tau
$$

\subsection{Chemical Reaction Controls Interfacial Progres- sion}

3.2.1. Description of Cylinder Height in Terms of Radius

Determining the relationship between the 'unique' radius, 'unique' height and the description of change in height and radius with degree of conversion is conducted in a manner similar to Eqs. (2) to (7).

$$
\begin{gathered}
\tau_{\text {i.c. }}=\frac{\rho_{\mathrm{B}} R_{\mathrm{u}}}{b k_{\mathrm{c}} C_{\mathrm{Ag}}} \\
\tau_{\text {f.p. }}=\frac{\rho_{\mathrm{B}} L_{\mathrm{u}}}{b k_{\mathrm{c}} C_{\mathrm{Ag}}}
\end{gathered}
$$

Where $k_{\mathrm{c}}$ is the chemical reaction rate constant.

For $\tau_{\text {i.c. }}=\tau_{\text {f.p. }}$

$$
L_{\mathrm{u}}=R_{\mathrm{u}}
$$

The fraction time of reduction for the flat plate and infinite cylinder are given by Levenspiel ${ }^{1)}$ as:

$$
\begin{aligned}
& \left(\frac{t}{\tau_{\text {i.c. }}}\right)=1-\frac{r}{R_{\mathrm{u}}} \\
& \left(\frac{t}{\tau_{\text {f.p. }}}\right)=1-\frac{l_{\mathrm{u}}}{L_{\mathrm{u}}}
\end{aligned}
$$

so for any given time $(t)$,

$$
l_{\mathrm{u}}=L_{\mathrm{u}} \cdot \frac{r}{R_{\mathrm{u}}}=f(r)
$$

For chemical reaction controlled conversion, the reaction velocity is constant at a rate $\left(k_{\mathrm{s}}\right)$, and as such, the height of the cylinder would change proportionally with radius.

Therefore we see that the geometric description given above satisfies this situation. To describe cases where $L \neq R$ Eq. (21) can be transposed.

$$
l=l_{\mathrm{u}}+L-L_{\mathrm{u}}=L_{\mathrm{u}}\left(\frac{r}{R}-1\right)+L
$$

On examining Eq. (22) we see that:

for $L>R$

$$
\tau_{\text {cyl }}=\tau_{\text {i.c. }}
$$

and that for $L<R$

$$
\tau_{\text {cyl }}=\tau_{\text {f.p. }}
$$

\subsubsection{Formulation Method of Model}

The description of rate of conversion of moles $\mathrm{B}$ with 
time is taken with respect to the reaction surface area. In the case of the shrinking core model, this area is that of the shrinking core (remembering that the cylinder height is $2 \cdot L)$ :

$$
\begin{aligned}
-\frac{d N_{\mathrm{B}}}{d t} & =-b \frac{d N_{\mathrm{A}}}{d t}=b k_{\mathrm{c}} A C_{\mathrm{Ag}} \\
& =2 \pi r\left(r+2 L_{\mathrm{u}}\left(\frac{r}{R}-1\right)+2 L\right) b k_{\mathrm{c}} C_{\mathrm{Ag}}
\end{aligned}
$$

It is possible to rewrite $N_{\mathrm{B}}$ in terms of the shrinking core.

$$
\begin{aligned}
& -2 \pi r\left(r+2 L_{\mathrm{u}}\left(\frac{r}{R}-1\right)-2 L\right) \cdot \rho_{\mathrm{B}} \frac{d r}{d t} \\
& =2 \pi r\left(r+2 L_{\mathrm{u}}\left(\frac{r}{R}-1\right)+2 L\right) b k_{\mathrm{c}} C_{\mathrm{Ag}} \\
& -\rho_{\mathrm{B}} \frac{d r}{d t}=b k_{\mathrm{c}} C_{\mathrm{Ag}}
\end{aligned}
$$

Now integrating

$$
\begin{gathered}
-\rho_{\mathrm{B}} \int_{R}^{r} d r=b k_{\mathrm{c}} C_{\mathrm{Ag}} \int_{0}^{t} d t \\
t \frac{b k_{\mathrm{c}} C_{\mathrm{Ag}}}{\rho_{\mathrm{B}}}=(R-r) \ldots .
\end{gathered}
$$

Note: Firstly the radius can be determined iteratively or explicitly from the degree of conversion $\left(X_{\mathrm{B}}\right)$ by the following correlation:

$$
\begin{aligned}
X_{\mathrm{B}}=\frac{V_{\text {total }}-V_{\text {core }}}{V_{\text {total }}} & =1-\frac{4 \pi \cdot r^{2}\left(L_{\mathrm{u}}\left(\frac{r}{R}-1\right)+L\right)}{4 \pi \cdot R^{2} L} \\
& =1-\frac{r^{2}\left(L_{\mathrm{u}}\left(\frac{r}{R}-1\right)+L\right)}{R^{2} L} \ldots \ldots . .
\end{aligned}
$$

and secondly, that for $L<R$ :

$$
r \neq 0 \quad \text { when } \quad t=\tau
$$

\subsection{Product Layer Diffusion Controls Interfacial Progression}

The method of analysis of diffusion controlled kinetics is to undertake a double integration. The first integration is that of the reactant concentration, or temperature for thermal diffusion, through the product layer, between the outer cylinder surface and the inner cylinder (un-reacted) surface. This is accomplished by using surface areas perpendicular to the reactant flux.

The second integration is that of the concentration profiles between the cylinders outer surface and the un-reacted core surface for time between $t=0$ and $t=\tau$, taking into account the moving un-reacted core surface.

3.3.1. Description of Cylinder Height in Terms of Radius Determining the relationship between the 'unique' ra- dius, 'unique' height and the description of change in height and radius with degree of conversion is conducted in a manner similar to Eqs. (2) to (7).

$$
\tau_{\text {i.c. }}=\frac{\rho_{\mathrm{B}} R_{\mathrm{u}}^{2}}{4 b D_{\mathrm{e}} C_{\mathrm{Ag}}}
$$

Where $D_{\mathrm{e}}$ is the product layer effective diffusion constant.

$$
\tau_{\text {f.p. }}=\frac{\rho_{\mathrm{B}} L_{\mathrm{u}}^{2}}{2 b D_{\mathrm{e}} C_{\mathrm{Ag}}}
$$

For $\tau_{\text {i.c. }}=\tau_{\text {f.p. }}$

$$
L_{\mathrm{u}}=\frac{R_{\mathrm{u}}}{\sqrt{2}}
$$

The fraction time of reduction for the flat plate and infinite cylinder are given by Levenspiel ${ }^{1)}$ as:

$$
\begin{aligned}
\left(\frac{t}{\tau_{\text {i.c. }}}\right)=1 & -\left(\frac{r_{\mathrm{u}}}{R_{\mathrm{u}}}\right)^{2}+\left(\frac{r_{\mathrm{u}}}{R_{\mathrm{u}}}\right)^{2} \cdot \ln \left(\left(\frac{r_{\mathrm{u}}}{R_{\mathrm{u}}}\right)^{2}\right) \ldots \ldots . . \\
& \left(\frac{t}{\tau_{\text {f.p. }}}\right)=\left(1-\frac{l_{\mathrm{u}}}{L_{\mathrm{u}}}\right)^{2} \ldots \ldots \ldots \ldots \ldots \ldots \ldots \ldots \ldots \ldots \ldots \ldots \ldots
\end{aligned}
$$

so for any given time $(t)$,

$$
\left(1-\frac{l_{\mathrm{u}}}{L_{\mathrm{u}}}\right)^{2}=1-\left(\frac{r_{\mathrm{u}}}{R_{\mathrm{u}}}\right)^{2}+\left(\frac{r_{\mathrm{u}}}{R_{\mathrm{u}}}\right)^{2} \cdot \ln \left(\left(\frac{r_{\mathrm{u}}}{R_{\mathrm{u}}}\right)^{2}\right) \ldots .
$$

solving Eq. (34) for $l_{\mathrm{u}}$ :

$$
l_{\mathrm{u}}=\frac{L_{\mathrm{u}}^{2}}{2}\left(\frac{2}{L_{\mathrm{u}}}-\frac{2}{R_{\mathrm{u}} \cdot L_{\mathrm{u}}} \cdot \sqrt{R_{\mathrm{u}}^{2}-r^{2}+r^{2} \cdot \ln \left(\frac{r^{2}}{R_{\mathrm{u}}^{2}}\right)}\right) \ldots \ldots
$$

or

$$
l_{\mathrm{u}}=f(r) .
$$

Schematically, the relationship of $l_{\mathrm{u}}$ and $r$ from Eq. (36) is shown in Fig. 6 for an arbitrary cylinder of radius $11 \mathrm{~mm}$ and height $\left(2 \cdot l_{\mathrm{u}}\right) 22 / \sqrt{2} \mathrm{~mm}$.

To describe cases where $L_{\mathrm{u}} \neq R_{\mathrm{u}} / \sqrt{2}$ Eq. (36) can be transposed.

$$
l=l_{\mathrm{u}}+L-L_{\mathrm{u}}
$$

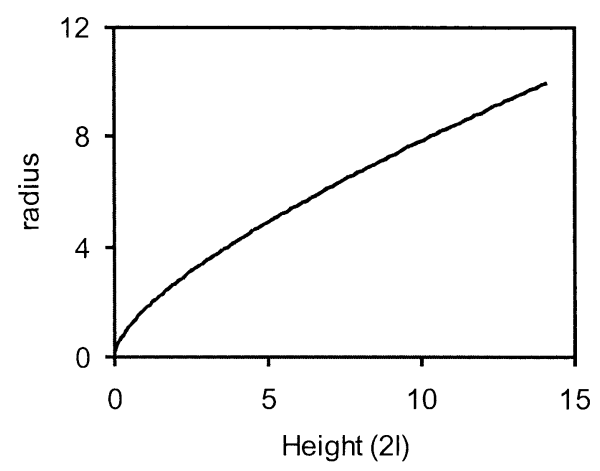

Fig. 6. Variation of cylinder height with radius for radius $=10$ and height $=20 / \sqrt{2}(\sim 14)$. 
On examining Eq. (37) we see that:

for $L<L_{\mathrm{u}}$,

$$
\tau_{\text {cyl }}=\tau_{\text {f.p. }}
$$

and that for $L>L_{\mathrm{u}}$,

$$
\tau_{\text {cyl }}=\tau_{\text {i.c. }}
$$

\subsubsection{Formulation Method of Model}

$$
\begin{array}{r}
-\frac{d N_{\mathrm{B}}}{d t}=-b \frac{d N_{\mathrm{A}}}{d t}=b A_{\mathrm{r}} D_{\mathrm{e}} \frac{d C_{\mathrm{A}}}{d r} \ldots \ldots \ldots \ldots . . . . . . . \\
-\frac{d N_{\mathrm{B}}}{d t}=2 \pi \cdot r\left(r+2\left(f(r)+L-L_{\mathrm{u}}\right)\right) b D_{\mathrm{e}} \frac{d C_{\mathrm{A}}}{d r} \ldots . \\
-\frac{d N_{\mathrm{B}}}{d t} \int_{R}^{r} \frac{d r}{r\left(r+2\left(f(r)+L-L_{\mathrm{u}}\right)\right)}=2 \pi \cdot b D_{\mathrm{e}} \int_{C_{\mathrm{Ag}}}^{0} d C_{\mathrm{A}} \ldots . .
\end{array}
$$

Rewriting the solution of the integration as $g(r)$ (a method for solving this is shown in Appendix A):

$$
g(r)=\int_{R}^{r_{\mathrm{c}}} \frac{d r}{r\left(r+2\left(f(r)+L-L_{\mathrm{u}}\right)\right)}
$$

We can then integrate for the span of time $=0$ to $\tau$.

$$
-\frac{d N_{\mathrm{A}}}{d t} g(r)=-2 \pi b D_{\mathrm{e}} C_{\mathrm{Ag}}
$$

It is possible to rewrite $N_{\mathrm{A}}$ in terms of the moving boundary of the shrinking core.

$$
2 \pi \rho_{\mathrm{B}} \int_{R}^{r_{\mathrm{c}}} g(r) \cdot r\left(r+2\left(f(r)+L-L_{\mathrm{u}}\right)\right) d r=2 \pi b D_{\mathrm{e}} C_{\mathrm{Ag}} \int_{0}^{t} d t
$$

The solution of Eq. (43) is rewritten in terms of $h(r)$ :

$$
h(r)=\int_{R}^{r_{\mathrm{c}}} g(r) \cdot r\left(r+2\left(f(r)+L-L_{\mathrm{u}}\right)\right) d r .
$$

so that an answer can be calculated from:

$$
t \frac{b D_{\mathrm{e}} C_{\mathrm{Ag}}}{\rho_{\mathrm{B}}}=h(r)
$$

Note: Firstly the radius can be determined iteratively from the degree of conversion $\left(X_{\mathrm{B}}\right)$ by the following correlation:

$$
\begin{aligned}
& X_{\mathrm{B}}=\frac{V_{\text {total }}-V_{\text {core }}}{V_{\text {total }}}=1-\frac{r^{2} \cdot l}{R^{2} \cdot L} \\
& =1-\frac{r^{2} \cdot\left(\frac { L _ { \mathrm { u } } ^ { 2 } } { 2 } \left(\frac{2}{L_{\mathrm{u}}}-\frac{2}{R_{\mathrm{u}} \cdot L_{\mathrm{u}}} \cdot \sqrt{\left.\left.R_{\mathrm{u}}^{2}-r^{2}+r^{2} \cdot \ln \left(\frac{r^{2}}{R_{\mathrm{u}}^{2}}\right)\right)+L-L_{\mathrm{u}}\right)}\right.\right.}{R^{2} \cdot L}
\end{aligned}
$$

Secondly, for $L<R / \sqrt{2}$ the limits for the radius range between $r=R$ and the iterative ' $r$ ' solution of $l-L+L_{\mathrm{u}}=f(r)$ for $l=0$ (i.e.: when the ends of the cylinder touch).

\subsection{Combination of Resistances}

As is often the case, more than one mechanism can control for a given scenario. Accordingly, it is important to be able to combine the models for each controlling mechanism. This is achieved by using the combination of resistances method.

$$
\text { Rate }=\frac{\text { Driving force }}{\text { Resistance }}
$$

In the case of fluid solid reactions, the driving force is the concentration gradient, and the resistance is everything else (including the rate constant). The models developed are shown in this form.

\subsubsection{Gas Film Diffusion}

$$
-\frac{d N_{\mathrm{B}}}{d t}=\frac{C_{\mathrm{Ag}}-C_{\mathrm{Ag}}^{\mathrm{eq} .}}{\left(\frac{1}{b k_{\mathrm{s}} A_{\text {external }}}\right)}
$$

\subsubsection{Chemical Reaction}

$$
-\frac{d N_{\mathrm{B}}}{d t}=\frac{C_{\mathrm{Ag}}-C_{\mathrm{Ag}}^{\mathrm{eq}}}{\left(\frac{1}{b k_{\mathrm{c}} A_{\text {core }}}\right)}
$$

\subsubsection{Product Layer Diffusion:}

$$
-\frac{d N_{\mathrm{B}}}{d t}=\frac{C_{\mathrm{Ag}}-C_{\mathrm{Ag}}^{\mathrm{eq}}}{\left(\frac{-g(r)}{2 \pi b D_{\mathrm{e}}}\right)}
$$

The overall rate equation becomes:

$$
-\frac{d N_{\mathrm{A}}}{d t}=\frac{\left(C_{\mathrm{Ag}}-C_{\mathrm{Ag}}^{\mathrm{eq} \cdot}\right)}{\frac{1}{\bar{k}}}=\bar{k}\left(C_{\mathrm{Ag}}-C_{\mathrm{Ag}}^{\mathrm{eq} \cdot}\right)
$$

where $\bar{k}$ is the averaged rate resistance and is determined by resistances in series, i.e.:

$$
\frac{1}{\bar{k}}\left(\frac{1}{b k_{\mathrm{s}} A_{\text {external }}}\right)+\left(\frac{1}{b k_{\mathrm{c}} A_{\text {core }}}\right)+\left(\frac{-g(r)}{2 \pi b D_{\mathrm{e}}}\right)
$$

so that the general rate equation becomes:

$$
-\frac{d N_{\mathrm{B}}}{d t}=\frac{\left(C_{\mathrm{Ag}}-C_{\mathrm{Ag}}^{\mathrm{eq}}\right)}{\left(\frac{1}{b k_{\mathrm{s}} A_{\text {external }}}\right)+\left(\frac{1}{b k_{\mathrm{c}} A_{\text {core }}}\right)+\left(\frac{-g(r)}{2 \pi b D_{\mathrm{e}}}\right)}
$$

\section{Results and Discussion}

\subsection{Kang et al. ${ }^{3)}$}

The data shown in Fig. 1 to Fig. 3 is manipulated according to Eqs. (10) to (45) and is displayed in Fig. 7 to Fig. 9.

As noted earlier, the experiments had been designed to minimise the effect of gas film diffusion on the overall reduction process ${ }^{3)}$ and it can be seen from Fig. 7 that gas film diffusion plays no part in controlling the overall reac- 


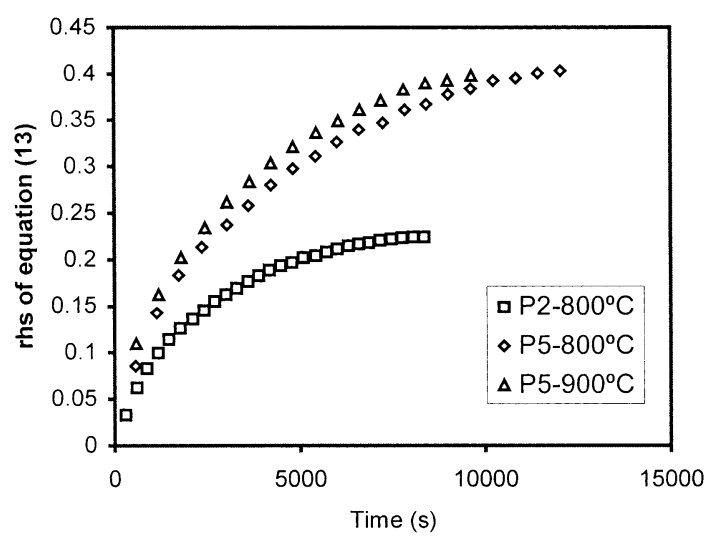

Fig. 7. Gas film diffusion equation.

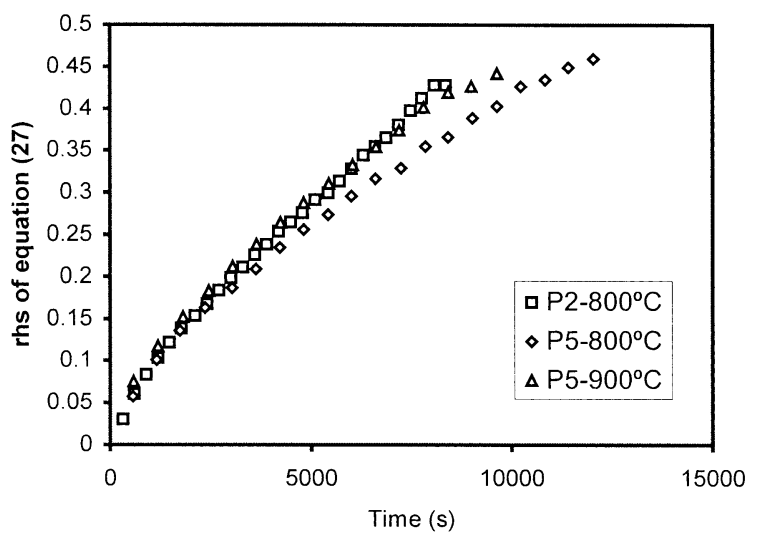

Fig. 8. Chemical Reaction Controlled Equation.

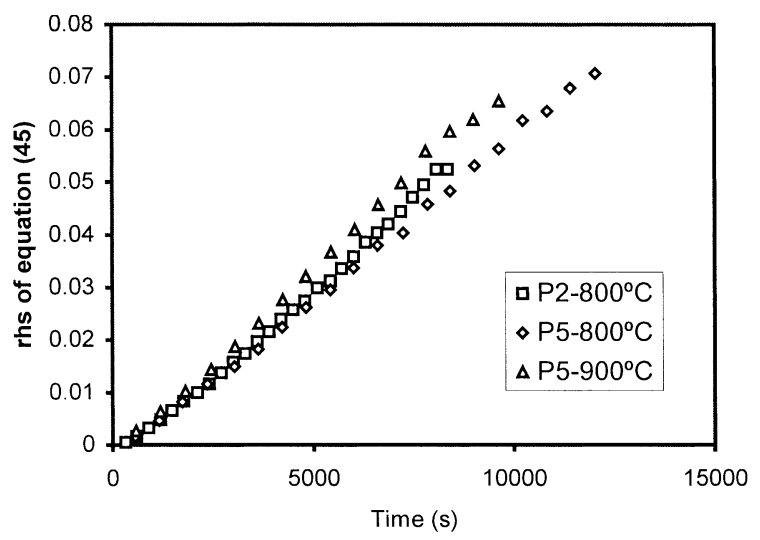

Fig. 9. Product layer Diffusion Controlled Equation.

tion.

Product layer diffusion (Fig. 9) displays the greatest degree of linearity, although there is some degree of linearity with chemical reaction (Fig. 8) which indicates mixed control for part or most of the reaction.

The method for determining the combined mechanism equation is shown in Eq. (52). In this case, it is the chemical reaction and product layer diffusion which control the reaction, so the contribution from the gas boundary layer can be ignored. Therefore, Eq. (52) becomes:

$$
-\frac{d N_{\mathrm{B}}}{d t}=\frac{\left(C_{\mathrm{Ag}}-C_{\mathrm{Ag}}^{\mathrm{eq}}\right)}{\left(\frac{1}{b k_{\mathrm{c}} A_{\text {core }}}\right)+\left(\frac{-g(r)}{2 \pi b D_{\mathrm{e}}}\right)}
$$

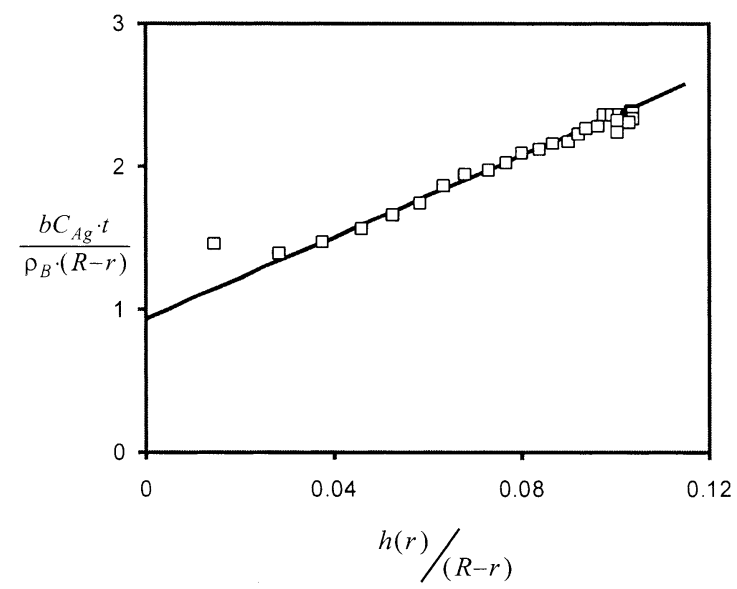

Fig. 10. Application of Eq. (59) to the data shown in Fig. 1 to determine $D_{\mathrm{e}}$ and $k_{\mathrm{c}}$ of $\mathrm{P} 2$ (see Table 1) at $800^{\circ} \mathrm{C}$.

Assuming a non-reversible reaction, then $C_{\mathrm{Ag}}^{\mathrm{eq}}=0$, and it is possible to rewrite $d N_{\mathrm{B}} / d t$ in terms of radius, therefore getting:

$$
-\left(\left(\frac{1}{b k_{\mathrm{c}} A_{\text {core }}}\right)+\left(\frac{-g(r)}{2 \pi b D_{\mathrm{e}}}\right)\right) A_{\text {core }} \frac{d r}{d t}=\frac{b C_{\mathrm{Ag}}}{\rho_{\mathrm{B}}} \ldots .
$$

For the integration step, an average value of un-reacted core radius and height are used. That is:

$$
r_{\text {combined }}=\frac{r_{\text {chem }}+r_{\text {ash }}}{2}
$$

where $r_{\text {chem }}$ and $r_{\text {ash }}$ are obtained from Eqs. (28) and (46) respectively and

$$
l_{\text {combined }}=\frac{l_{\text {chem }}+l_{\text {ash }}}{2}
$$

where $l_{\text {chem }}$ and $l_{\text {ash }}$ are determined from Eqs. (22) and (37) respectively.

So that Eq. (54) becomes:

$$
\begin{aligned}
& -\frac{1}{k_{\mathrm{c}}} \int_{R}^{r} d r-\frac{1}{D_{\mathrm{e}}} \int_{R}^{r}-g(r) \cdot r(r+2 l) d r=\frac{b C_{\mathrm{Ag}}}{\rho_{\mathrm{B}}} \int_{0}^{t} d t \\
& \frac{1}{k_{\mathrm{c}}}(R-r)+\frac{1}{D_{\mathrm{e}}}(h(r))=\frac{b C_{\mathrm{Ag}}}{\rho_{\mathrm{B}}} t
\end{aligned}
$$

Kang et al. ${ }^{3)}$ show a method of determining the constants $k_{\mathrm{c}}$ and $D_{\mathrm{e}}$ by rearranging Eq. (58).

$$
\frac{b C_{\mathrm{Ag}}}{\rho_{\mathrm{B}} \cdot(R-r)} t=\frac{1}{D_{\mathrm{e}}} \frac{(h(r))}{(R-r)}+\frac{1}{k_{\mathrm{c}}}
$$

or

$$
y=m \cdot x+c
$$

The plots for this relationship are shown in Fig. 10 to Fig. 12.

The kinetic values obtained from Fig. 10 to Fig. 12 (Kang et al.) and Fig. 14 (Nasr et al.) are given in Table 2. The kinetic values obtained here are similar to those obtained by Kang et al. ${ }^{3)}$ (shown in Table 3) for all geometries studied. Unfortunately comparative data from Nasr et 


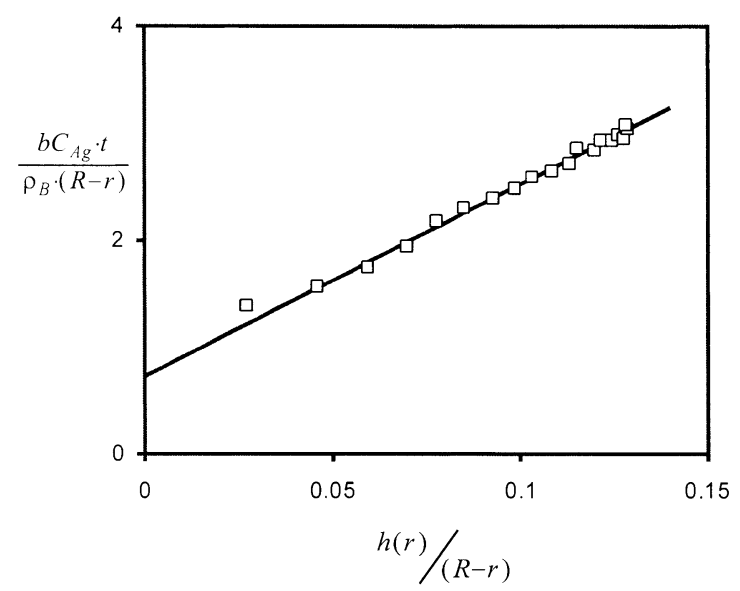

Fig. 11. Application of Eq. (59) to the data shown in Fig. 2 to determine $D_{\mathrm{e}}$ and $k_{\mathrm{c}}$ of P5 (see Table 1) at $800^{\circ} \mathrm{C}$.

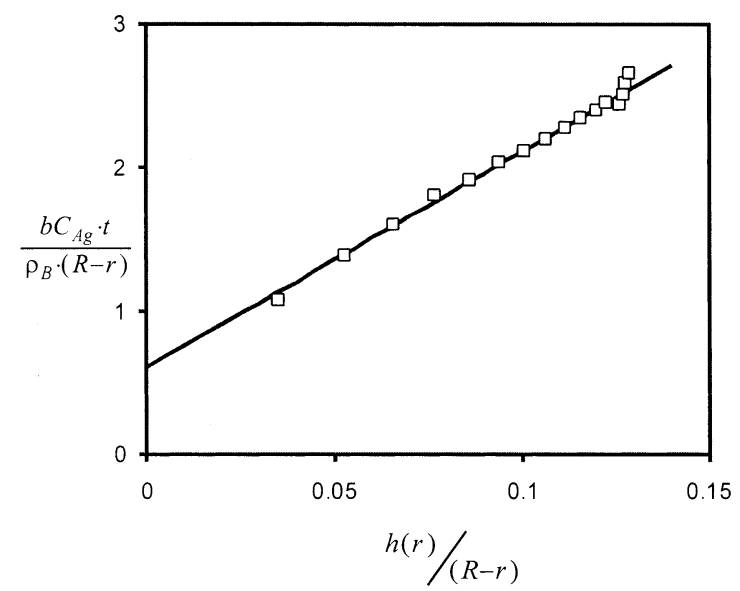

Fig. 12. Application of Eq. (59) to the data shown in Fig. 3 to determine $D_{\mathrm{e}}$ and $k_{\mathrm{c}}$ of P5 (see Table 1) at $900^{\circ} \mathrm{C}$.

Table 2. Kinetic values for finite cylinders.

\begin{tabular}{|c|c|c|c|c|}
\hline Temp. & Dimensions & Author ID & $k_{c}(\mathrm{~cm} / \mathrm{s})$ & $D_{e}\left(\mathrm{~cm}^{2} / \mathrm{s}^{\prime}\right)$ \\
\hline $800^{\circ} \mathrm{C}$ & $\phi 1.19 \mathrm{~cm} \times 0.9 \mathrm{~cm}$ & P2 - Kang et al3' & 1.07 & 0.07 \\
\hline $800^{\circ} \mathrm{C}$ & $\phi 1.66 \mathrm{~cm} \times 0.92 \mathrm{~cm}$ & P5 - Kang et al $3^{\prime}$ & 1.37 & 0.06 \\
\hline $900^{\circ} \mathrm{C}$ & $\phi 1.66 \mathrm{~cm} \times 0.92 \mathrm{~cm}$ & P5 - Kang et al $3^{\prime}$ & 1.64 & 0.07 \\
\hline $800^{\circ} \mathrm{C}$ & $\phi 0.79 \mathrm{~cm} \times 0.835 \mathrm{~cm}$ & Nasr et al4 & 0.37 & - \\
\hline
\end{tabular}

Table 3. Kinetic data obtained from Kang et al. . $^{3)}$

\begin{tabular}{|c|c|c|c|c|c|}
\hline Temp. & Dimensions & Geometry & Author ID & $k_{c}(\mathrm{~cm} / \mathrm{s})$ & $D_{e}\left(\mathrm{~cm}^{2} / \mathrm{s}\right)$ \\
\hline $800^{\circ} \mathrm{C}$ & $\phi 1.24 \mathrm{~cm}$ & Sphere & P1 & 1.37 & 0.057 \\
\hline $900^{\circ} \mathrm{C}$ & $\phi 1.24 \mathrm{~cm}$ & Sphere & P1 & 2.45 & 0.068 \\
\hline $800^{\circ} \mathrm{C}$ & $\phi 1.19 \mathrm{~cm} \times 0.9 \mathrm{~cm}$ & Cylinder & P2 & 1.26 & 0.052 \\
\hline $900^{\circ} \mathrm{C}$ & $\phi 1.19 \mathrm{~cm} \times 0.9 \mathrm{~cm}$ & Cylinder & P2 & 2.21 & 0.067 \\
\hline $800^{\circ} \mathrm{C}$ & $\phi 1.56 \mathrm{~cm}$ & Sphere & P4 & 1.73 & 0.055 \\
\hline $900^{\circ} \mathrm{C}$ & $\phi 1.56 \mathrm{~cm}$ & Sphere & P4 & 3.36 & 0.068 \\
\hline $800^{\circ} \mathrm{C}$ & $\phi 1.66 \mathrm{~cm} \times 0.92 \mathrm{~cm}$ & Cylinder & P5 & 1.38 & 0.048 \\
\hline $900^{\circ} \mathrm{C}$ & $\phi 1.66 \mathrm{~cm} \times 0.92 \mathrm{~cm}$ & Cylinder & P5 & 2.68 & 0.064 \\
\hline
\end{tabular}

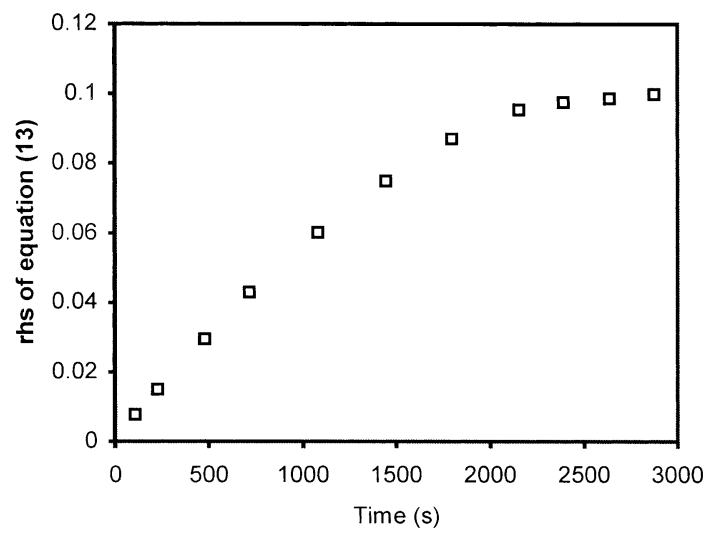

Fig. 13. Gas film diffusion equation.

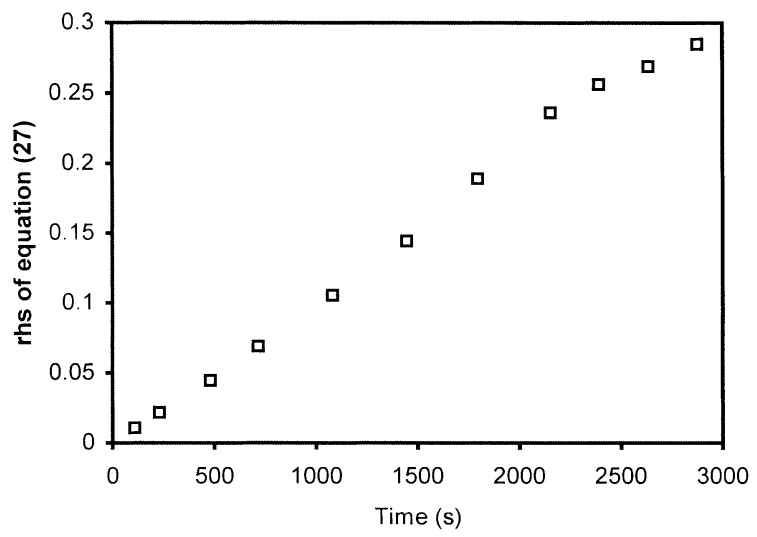

Fig. 14. Chemical Reaction Controlled Equation.

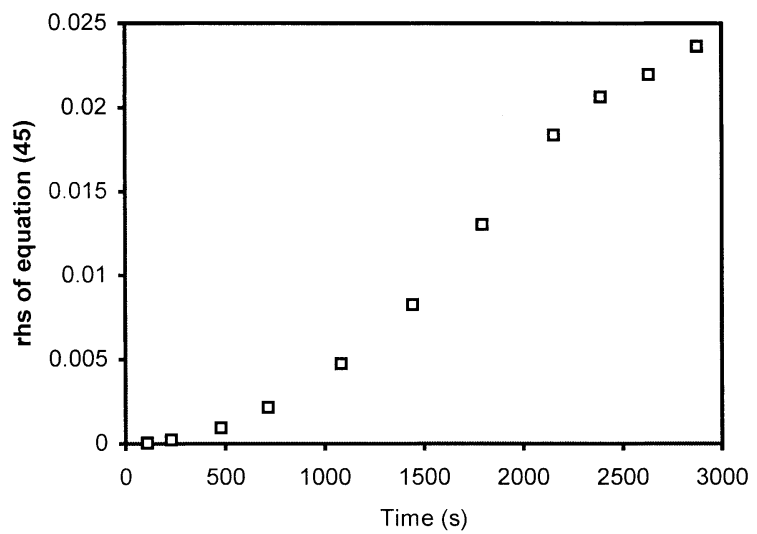

Fig. 15. Product layer Diffusion Controlled Equation.

al. ${ }^{4)}$ was not available.

\subsection{Nasr et al. ${ }^{4)}$}

The data shown in Fig. 4 is manipulated according to Eqs. (10) to (45) and is displayed in Fig. 13 to Fig. 15.

It can be seen that the chemical reaction displays the greatest degree of linearity, and hence inferred control) of the three mechanisms. This is not surprising considering the small size of the sample. The rate constant $k_{\mathrm{c}}$ is much lower than that of Kang et l. $^{3)}$ This is due to the reaction system being that of $\mathrm{Fe}_{2} \mathrm{O}_{3}$ to $\mathrm{Fe}$, which includes the slow $\mathrm{Fe}_{\mathrm{t}} \mathrm{O}$ to Fe step. ${ }^{6}$ 


\section{Conclusions}

A series of methods have been developed to describe the shrinking core model conversion of a finite cylinder for gas film diffusion, chemical reaction and product layer diffusion controlled mechanisms.

The models have been validated using data from literature and have yielded kinetic data similar to that from literature.

\section{REFERENCES}

1) O. Levenspiel: Chemical Reaction Engineering, 2nd Ed., Wiley, New York, (1972), 360.

2) A. N. Gorkan and L. K. Doraiswamy: Chem. Eng. Sci., 3 (1973), 401.

3) H. W. Kang, W. S. Chung, T. Murayama and Y. Ono: ISIJ Int., 38 (1998), 324.

4) M. I. Nasr, A. A. Omar, M. M. Hessien and A. A. El-Geassy: ISIJ Int., 36 (1996), 164.

5) H. W. Kang, W. S. Chung and T. Murayama: ISIJ Int., 38 (1998), 109.

6) V. Sahajwalla, J. M. Cadogan and A. Zhou: The AusIMM Annual Conf., AusIMM, Parkville, (1996), 207.

\section{Appendix}

Method for solving of $g(r) . g(r)$ is defined as:

$$
\begin{aligned}
g(r)= & \int_{R}^{r_{\mathrm{c}}}\left\{r \left(r+2\left(\frac { L _ { \mathrm { u } } ^ { 2 } } { 2 } \left(\frac{2}{L_{\mathrm{u}}}-\frac{2}{R_{\mathrm{u}} \cdot L_{\mathrm{u}}}\right.\right.\right.\right. \\
& \cdot \sqrt{\left.\left.\left.\left.R_{\mathrm{u}}^{2}-r^{2}+r^{2} \cdot \ln \left(\frac{r^{2}}{R_{u}^{2}}\right)\right)+L-L_{\mathrm{u}}\right)\right)\right\}^{-1} d r}
\end{aligned}
$$

Due to the complexity of the integral $g(r)$, one method for obtaining a solution is to take a Taylor Series of the integrand. A Taylor Series can be described as:

$$
\begin{aligned}
g(r)= & f(a)+f^{\prime}(a)(r-a) \\
& +\frac{f^{\prime \prime}(a)}{2 !}(r-a)^{2}+\cdots+\frac{f^{\prime \prime}(a)}{n !}(r-a)^{n}+\cdots
\end{aligned}
$$

The order $(n)$ of the expansion is taken as that which gives an acceptable degree of error (see Table A1). The advantage of using a Taylor series is in its ease of integratability
Table A1. Maximum relative error for increasing order $(n)$.

\begin{tabular}{|c|c|c|}
\hline $\mathrm{n}$ & \% Error at $r=2.9 \mathrm{~mm}$ & $\%$ Error at $r=R=10 \mathrm{~mm}$ \\
\hline 5 & 314 & 25 \\
\hline 10 & 118 & 9 \\
\hline 20 & 16 & 0.9 \\
\hline 40 & 0.26 & 0.009 \\
\hline 50 & 0.03 & 0.0009 \\
\hline
\end{tabular}

as compared to the original function $g(r)$. Although some functions have an infinite number of terms to describe them from to $-\infty, \infty$ we are only concerned with the range of $R$ to $r$. The expansion point ( $a$ ) chosen is the mid-point of the radial limits $(R+r) / 2$. An example of a first order Taylor Series expansion about $(R+r) / 2$ is shown below:

$$
\begin{aligned}
& g(r) \approx \frac{1}{a\left[a+L_{\mathrm{u}}^{2}\left[\frac{2}{L_{\mathrm{u}}}-\frac{2}{R L_{\mathrm{u}}} \sqrt{\left.\left.R^{2}-a^{2}+a^{2} \ln \left(\frac{a^{2}}{R^{2}}\right)\right]+2 L-2 L_{\mathrm{u}}\right]}\right.\right.} \\
& +\left[\frac{-1}{a^{2}}+\frac{R}{a\left(a R-2 L_{\mathrm{u}} \sqrt{R^{2}-a^{2}+a^{2} \ln \left(\frac{a^{2}}{R^{2}}\right)-2 L R}\right)}\right. \\
& \left.\left[1-\frac{2 a L_{\mathrm{u}} \cdot \ln \left(\frac{a^{2}}{R^{2}}\right)}{R \cdot \sqrt{R^{2}-a^{2}+a^{2} \ln \left(\frac{a^{2}}{R^{2}}\right)}}\right]\right] \\
& \cdot\left[a+L_{\mathrm{u}}^{2}\left[\frac{2}{L_{\mathrm{u}}}-\frac{2}{R L_{\mathrm{u}}} \sqrt{R^{2}-a^{2}+a^{2} \ln \left(\frac{a^{2}}{R^{2}}\right)}\right]+2 L-2 L_{\mathrm{u}}\right]^{-1} \\
& \cdot(r-a)
\end{aligned}
$$

The maximum percentage error (occurring at $r=0$ and $r=R$ ) of the Taylor Expansion at $a=6.5 \mathrm{~mm}$ and Height $=$ $12 \mathrm{~mm}$ over a nominal radial range of $R=10 \mathrm{~mm}$ to $l(r)=0$ $(r=2.9 \mathrm{~mm})$ is shown in Table A1. 